\title{
Erratum to: Transoral Robotic Resection of Parapharyngeal Space Tumors
}

\author{
Moran Amit, Irit Duek, and Ziv Gil
}

\section{Erratum to: \\ Chapter 13 in: Z. Gil et al. (eds.), Atlas of Head and Neck Robotic Surgery, DOI 10.1007/978-3-319-49578-1_13}

The second author's name had been incorrectly spelt and the same has been corrected. The author's full name is Irit Duek.

The updated online version of this chapter can be found at DOI 10.1007/978-3-319-49578-1_13

M. Amit, MD, PhD

The Laboratory for Applied Cancer Research, Department of Otolaryngology Head and Neck Surgery, the Head and Neck Center, Rambam Healthcare Campus, Clinical Research Institute at Rambam, Rappaport Institute of Medicine and Research, The Technion, Israel Institute of Technology, Haifa, Israel

I. Duek, MD

Department of Otolaryngology Head and Neck Surgery, the Head and Neck Center, Rambam Healthcare Campus, Clinical Research Institute at Rambam, Rappaport Institute of Medicine and Research, The Technion, Israel Institute of Technology, Haifa, Israel

Z. Gil, MD, PhD $(\bowtie)$

The Laboratory for Applied Cancer Research, Department of Otolaryngology Head and Neck Surgery, the Head and Neck Center, Rambam Healthcare Campus, Clinical Research Institute at Rambam, Rappaport Institute of Medicine and Research, The Technion, Israel Institute of Technology, Haifa, Israel

Department of Otolaryngology, Rambam Medical Center, The Technion, Israel Institute of Technology, Haifa 66036, Israel e-mail: ziv@baseofskull.org 